\title{
Angle-closure Glaucoma: treatment
}

\author{
Glaucoma de Ângulo fechado: Tratamento \\ Authorship: Brazilian Council of Ophthalmology \\ Participants: Prata TS, Kanadani FN, Simões R, Bernardo WM
}

http://dx.doi.org/10.1590/1806-9282.60.04.004

The Guidelines Project, an initiative of the Brazilian Medical Association, aims to combine information from the medical field in order to standardize procedures to assist the reasoning and decision-making of doctors.

The information provided through this project must be assessed and criticized by the physician responsible for the conduct that will be adopted, depending on the conditions and the clinical status of each patient.

\section{Description OF THE EVIDENCE COLLECTION METHOD}

The literature review of scientific articles in this guideline was held in the databases Medline, Cochrane and SciELO. The search for evidence came from actual clinical scenarios and used keywords (MeSH terms) grouped in the following syntax: (glaucoma, angle-closure or glaucoma, closed angle or glaucomas, closed-angle or glaucoma, uncompensative or glaucomas, uncompensative or angle closure glaucoma or angle closure glaucomas or glaucomas, angle closure or glaucoma, narrow-angle) and (iridectomy or corectomy or corectomies or laser therapy). The articles were selected after critical evaluation of the strength of scientific evidence, and publications of greatest strength were used for recommendation. The recommendations were drawn from group discussion. The entire guideline was reviewed by an independent group specializing in evidence-based clinical guidelines.

\section{DEGREE OF RECOMMENDATION AND STRENGTH OF EVIDENCE}

A: Experimental or observational studies of higher consistency.

B: Experimental or observational studies of lower consistency.

C: Case reports (non-controlled studies).

D: Opinions without critical evaluation, based on consensus, physiological studies, or animal models.

\section{Objective}

To evaluate the indication of laser iridotomy in patients with established or suspected primary angle-closure glaucoma.

\section{Conflict of interest}

No conflict of interest informed.

\section{INTRODUCTION}

The prevalence of glaucoma varies with age and ethnic origin, and for Caucasians in the age range of approximately 45 years it is between $2.1 \%$ and $3 \%$ in primary open-angle glaucoma and at $0.3 \%$ in primary angle-closure glaucoma. By the age of 55 years, it reaches between $2.2 \%$ and $5 \%$ in Caucasians, and may even amounts to $47.8 \%$ among Vietnamese in the same age group. In the world as a whole, however, angle-closure glaucoma is the leader among the diseases that cause blindness ${ }^{1}(\mathbf{D})$.

The process of angle closure is characterized by partial or complete closure of the anterior chamber angle due to anatomic conditions that promote apposition or adhesion of the peripheral iris tissue to its external wall, with frequent rise in intraocular pressure (IOP). IOP above normal limits can lead to glaucomatous optic neuropathy and threaten the sight in two ways: acute primary angle closure (previously called acute glaucoma) and primary angle-closure glaucoma (chronic form of the disease) $)^{2,3}(\mathbf{D})$. It is important to know the epidemiological and ocular factors that predispose to angle closure and consequently to primary angle-closure glaucoma: female gender, advanced age, eastern Asian ascendance, narrow angle, shallow anterior chamber, reduced corneal diameter, smaller corneal radius of curvature, eyes with reduced axial diameter and anterior displacement/increased anteroposterior diameter of the lens ${ }^{3}(\mathbf{D})^{4,5}(\mathbf{C})$.

\section{IN A PATIENT WITH ACUTE PRIMARY ANGLE CLOSURE IN ONE EYE, WOULD A PROPHYLACTIC LASER IRIDOTOMY IN THE CONTRALATERAL EYE BE INDICATED?}

The Inuit of Greenland have a shallower anterior chamber already described in world populations and the proportion of blindness from angle-closure glaucoma was 
reduced from $64 \%$ to $9 \%$ within 37 years through the adoption of prophylactic measures such as measuring the depth of the anterior chamber, Van Herick testing, gonioscopy, surgical iridectomy or laser iridotomy ${ }^{6}(\mathbf{D})$.

After an episode of acute primary angle closure, performing iridotomy in the contralateral eye can prevent similar episodes in many cases, and increased intraocular pressure (IOP) occurs in approximately $12 \%$ of patients treated, compared to $75 \%$ in eyes not $\operatorname{treated}^{7}(\mathbf{A})^{8-10}(\mathbf{C})$.

A retrospective study conducted in Singapore included subjects with a mean age of 64 years (being $87.5 \%$ Chinese) with acute primary angle closure attacks (defined as the presence of at least two of the following symptoms: periocular pain, nausea, vomiting or both; presence of IOP above $21 \mathrm{mmHg}$ and associated with at least three of the following signs: conjunctival injection, edema of the corneal epithelium, non-reactive pupil, and the presence of occludable angle on gonioscopy). In this study, the authors found that after prophylactic treatment with laser peripheral iridotomy (LPI), 71 contralateral eyes (corresponding to $88.8 \%$ of the eyes treated with LPI) showed good IOP control in the long term, without the need of additional treatment for the glaucoma and with no reports of new attacks (mean follow-up of approximately 51 months) ${ }^{9}$ (C).

Nevertheless, controversial results were reported in a retrospective study by Chen et al, which analyzed individuals (mean age 71.8 years) with acute primary angle closure attacks treated with LPI and Nd:YAG laser, finding high frequency of IOP elevation ( $92.6 \%$ of eyes) during a median follow-up period of 36 months. In this study, approximately $52 \%$ of cases needed further treatment to control pressure ${ }^{11}(\mathbf{C})$. A recent study by Rao et al. suggests that the IOP at the time of presentation (before laser treatment) is an important predictor of the success or failure of the procedure in terms of pressure control in the medium-long term ${ }^{12}(\mathbf{C})$.

In this case, those with higher IOP (an indirect measure of the functional reserve of the trabecular meshwork) would have a greater chance of needing additional treatment to control IOP.

There are reports stating that LPI is as effective as surgical iridectomy although being non-invasive, having lower cost, being more convenient and $\operatorname{safe}^{13,14}(\mathbf{D})$. It is thus more indicated as prophylaxis ${ }^{15,16}(\mathbf{A})$.

The complications described include transient bleeding at the site of laser iridotomy, acute increase in IOP, anterior uveitis, formation of posterior synechia, possible acceleration of the development of cataracts (controversial), corneal decompensation and ciliochoroidal effusion ${ }^{17}(\mathbf{B})^{18}(\mathbf{C})$.

\section{Recommendation}

Prophylactic laser peripheral iridotomy must be considered in patients with primary angle closure in one eye and history of acute primary angle closure (acute attack of angle-closure glaucoma) in the other. In these cases, the laser iridotomy should be preferred over surgical iridectomy. This would be indicated in cases where the first cannot be done.

\section{IN WHICH ANATOMIC SITUATION OF THE CAMERULAR SINUS IS THERE INDICATION OF PROPHYLACTIC LASER IRIDOTOMY?}

During routine eye examination, the presence of peripheral anterior synechia may be indicative of primary angle closure. In these cases there is evidence that, if performed early, prophylactic laser iridotomy can help in the prevention of acute primary angle closure (acute attack of angle-closure glaucoma), with direct influence on the deepening of the anterior chamber ${ }^{6}(\mathbf{D})^{19-21}(\mathbf{C})$.

In previous population studies, it was shown that the epidemiological definition of narrow angle or possible occlusion (occludable) is inaccurate. A practical definition that is more often adopted today would be that any eye in which the posterior trabecular meshwork (pigmented portion of the trabecular meshwork) may not be seen on gonioscopy at 180 degrees or more, without additional maneuvers, in primary position of gaze, in dark room conditions, using narrow slit lamp beam, and ensuring that no light falls on the pupil to avoid opening of angle $e^{2,3}(\mathbf{D})$.

Currently, studies have reported the primary angle-closure glaucoma as an ongoing process, suggesting a continuum of the disease, which begins with angle closure and culminates with the development of glaucomatous optic neuropathy. This classification, based on the evolution of the disease, is divided into three $\operatorname{stages}^{2}(\mathbf{D})$. The stage of disease and other individual characteristics determine whether prophylactic laser peripheral iridotomy is indicated.

- Suspected primary angle closure: when the patient has only occludable angle (see paragraph above for definition).

- Primary angle closure: when the patient already presents signs of the process of primary angle closure: imprint and/or goniosynechia or increased IOP.

- Primary angle-closure glaucoma: when the patient shows signs of glaucomatous optic neuropathy and/ or visual field defect consistent with glaucoma.

In patients with primary angle closure ( $2^{\text {nd }}$ stage), prophylactic laser peripheral iridotomy is formally indicated. In 
the $1^{\text {st }}$ stage, when patients still do not show signs of primary angle closure (normal IOP and absence of goniosynechia/imprint), the decision whether to perform prophylactic laser iridotomy will also depend on the analysis of some important individual factors that are not in the described classification, such as presence of symptoms, family history of glaucoma or blindness, patient follow-up capacity, patient's socioeconomic conditions, and frequent need of pupillary dilation. It is noteworthy that in these cases ( $1^{\text {st }}$ stage) literature does not provide much support and little is known of the natural history of these patients. In addition, there is a lack of randomized clinical trials comparing prophylactic laser iridotomy versus a control group $\mathrm{p}^{22}(\mathbf{B})^{23}(\mathbf{C})$. If the choice is that of not performing the laser procedure, clinical observation of the iridocorneal angle and the IOP in routine visits will be important $\mathrm{t}^{2}(\mathbf{D})$. The onset of symptoms or signs of angle closure identified upon gonioscopic evaluation constitute an absolute indication for iridectomy.

\section{Recommendation}

Prophylactic laser iridotomy is indicated in every case diagnosed as primary angle closure (patients without glaucoma, but with imprint and/or goniosynechia or increased IOP). In cases of suspected primary angle closure (occludable angle, but normal IOP and absence of goniosynechia and/or imprint), the medical literature does not yet provide consensus, being the prophylactic laser procedure indicated or not, depending on the factors previously discussed.

\section{References}

1. Foster PJ, Johnson GJ. Glaucoma in China how big is the problem? Br J Ophthalmol 2001;85:1277-82.

2. Foster PJ. The epidemiology of primary angle closure and associated glaucomatous optic neuropathy. Semin Ophthalmol 2002;05:50 PM-8.
3. Vasconcelos JPC, Sakata LM, Silva MJL e Costa VP. $2^{\circ}$ Consenso de Glaucoma Primário de Ângulo Fechado. Sociedade Brasileira de Glaucoma, 2012.

4. Lowe RF. The second eye: an analysis of 200 cases. Br J Ophthalmol 1962; 45:641-50.

5. Sheth HG, Goel R, Jain S. UK national survey of prophylactic YAG iridotomy. Eye 2005;19.981-4.

6. Johnson GJ, Foster PJ. Can we prevent angle-closure glaucoma? Eye 2005; 19:1119-24.

7. Saw SM, Gazzard G, Friedman DS. Interventions for Angle-Closure Glaucoma. An Evidence-Based Update. Opththalmology 2003;110:10869-1879.

8. Snow JT. Value of prophylactic peripheral iridectomy on the second eye in angle-closure glaucoma. Trans Ophthalmol Soc UK 1977;97:189-91.

9. Ang LP, Aung T, Chew PT. Acute primary angle closure in an Asian population: long-term outcome of the fellow eye after prophylactic laser peripheral iridotomy. Ophthalmology 2000;107:2092-6.

10. Lowe RF. Acute Angle-closure glaucoma - the second eye: ananalysis of 200 cases. Br J Ophthalmol 1962;46:641.

11. Chen MJ, Cheng CY, Chou CK, Liu CJ, Hsu WM. The long-term effect of Nd:YAG laser iridotomy on intraocular pressure in Taiwanese eyes with primary angle-closure glaucoma. J Chin Med Assoc. 2008;71:300-4.

12. Rao A, Rao HL, Kumar AU, Babu JG, Madhulata U, Arthi J, Tukaram M, Senthil S, Garudadri CS. Outcomes of laser peripheral iridotomy in angle closure disease. Semin Ophthalmol 2013;28:4-8.

13. Rich W. The economics of Nd-YAG laser treatment. Dev Ophthalmol. 1987;14:93-6.

14. Spaeth GL. Laser treatment is invasive surgery. Ophthalmic Surg. 1988;19:463-4.

15. Flerck BW, Wright E, Fairley EA. A randomised prospective comparison of operative peripheral iridectomy and Nd:YAG laser iridotomy treatment of acute angle closure glaucoma: 3 year visual acuity and intraocular. $\mathrm{Br} \mathrm{J}$ Ophthalmol 1997;81:884-8.

16. Schwenn O, Sell F. Prophylactic Nd:YAG-laser iridotomy versus surgical iridectomy: a randomised, prospective study. Ger J Ophthalmol 1995;4:374-9.

17. Sakai H, Ishikawa H, Shinzato M, Nakamura Y, Sakai M, Sawaguchi S. Prevalence of ciliochoroidal effusion after prophylactic laser iridotomy. Am J Ophthalmol 2003;136:537-8.

18. Marraffa M, Marchini G, Pagliarusco A, Perfetti S, Toscano A, Brunelli C, et al. Ultrasound biomicroscopy and corneal endothelium in Nd:YAG-laser iridotomy. Ophthalmic Surg Lasers 1995;26:519-23.

19. Lim LS, Aung T, Husain R, Wu YJ, Gazzard G, Seah SK. Acute primary angle closure: configuration of the drainage angle in the first year after laser peripheral iridotomy. Ophthalmology 2004;111:1470-4.

20. Foster PJ, Aung T, Nolan WP, Machin D, Baasanhu J, Khaw PT, et al. Defining 'occludable' angles in population surveys: drainage angle width, peripheral anterior synechia and glaucomatous optic neuropathy in east Asian people. Br J Ophthalmol 2004;88:486-90.

21. Nolan WP, Foster PJ, Devereux JG, Uranchimeg D, Johnson GJ, Baasanhu J. YAG laser iridotomy treatment for primary angle-closure in East Asian eyes. Br J Ophthalmol 2000;84:1255-9.

22. Thomas R, George R, Parikh R, Muliyil J, Jacob A. Five year risk of progression of primary angle closure suspects to primary angle closure: a population based study. Br J Ophthalmol 2003;87:450-4.

23. Wilensky JT, Kaufman PL, Frohlichstein D, Gieser DK, Kass MA, Ritch R, et al. Follow-up of angle-closure glaucoma suspects. Am J Ophthalmol 1993;115:338-46. 